\title{
Crystallization Behavior of Semi-Flexible Liquid Crystalline Polyesters and Their Blends
}

\author{
Young Deuk Yoo and Sung Chul KIM* \\ Department of Chemical Engineering, \\ Korea Advanced Institute of Science and Technology, \\ P. O. Box 131, Dongdaemun, Seoul, Korea
}

(Received March 25, 1988)

\begin{abstract}
The crystallization behavior of semi-flexible liquid crystalline polyesters and their blends has been investigated by differential scanning calorimetry and optical microscopy. The Avrami exponent of the nematic polyester having hexamethylene spacer (NP6) is about 2 which suggests rod-like growth from sporadic nuclei, and the solid structure of the polymer is very similar to that of the melt state. However, the nematic and smectic polyesters having decamethylene spacer (NP10, SP10) show gross recognization of the structure during crystallization. The length of the methylene flexible spacer has a significant effect on the crystallization behavior of liquid crystalline polymers. In blends of nematic and smectic polymers, the crystallization rate of the smectic component (SP10) is accelerated by the addition of small amounts of nematic polymers (NP6, NP10) which may act as heterogeneous nuclei, while that of the nematic component is slowed as the concentration of the smectic component increases.

KEY WORDS Crystallization / Kinetics / Semiflexible / Liquid Crystalline Polymer / Avrami / Blend / Nucleation / Isothermal /
\end{abstract}

There have been a few investigations on the crystallization behavior of low molecular weight liquid crystalline materials. Adamski and coworkers $^{1,2}$ have studied the transition of the cholesteryl pelargonate and caproate from the liquid crystal state to the solid crystalline state. Price et al..$^{3-5}$ studied the crystallization of several cholesteryl esters. They obtained information on the crystal growth geometry from the Avrami type equation. Contrary to these low molecular weight mesogens, very little research has been conducted on the crystallization kinetics of the liquid crystalline polymers, which has to be investigated in order to analyze the effects of the processing parameters on product properties.

Warner and Jaffe ${ }^{6}$ studied the crystallization behavior of a number of thermotropic nematic polyesters having rigid backbones by using the
Avrami equation. They found that massive structural rearrangements do not occur during crystallization and the structure of the solid crystal closely resembles that of the liquid crystal. But this quiescent crystallization may not be expected for liquid crystalline polymers having a flexible spacer in the main chain because of the relatively large amount of entropy change upon crystallization owing to the crystallizable flexible spacers. No investigations on the crystallization kinetics of the semi-flexible liquid crystalline polymers have been reported in the literature. The crystalization of the nematic melt is expected to occur rapidly compared to that of the isotropic melt, but different crystallization behavior is expected for the smectic melt because of both the compact solid-like structure and the highly viscous nature of the smectic phase.

\footnotetext{
* To whom all correspondence should be addressed.
} 
In the present study, the crystallization kinetics of the nematic and smectic liquid crystalline polyesters containing polymethylene flexible spacer in the main chain and their blends were investigated by the isothermal DSC method and microscopic observation. The Avrami analysis was undertaken to obtain the information on the crystal growth geometry and factors controlling the rate of crystallization.

\section{EXPERIMENTAL}

\section{Materials}

Two different nematic polyesters, NP6 and NP10, and one smectic polyester (smectic A), SP10, were selected for the present study. The preparation method and properties of the polymers were reported earlier by Lenz et al. $^{7-10}$

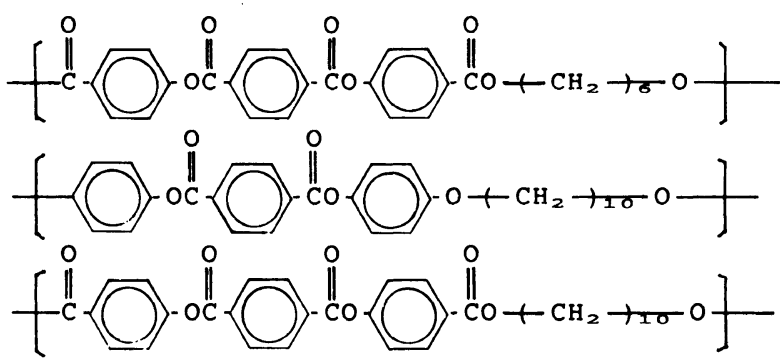

Two types of blends were prepared from the polymers, NP6-SP10 and NP10-SP10, using solution/precipitation method. The former contained two components which differed in the length of flexible segment, while the latter contained those which differed in the rigid mesogenic group. The detailed preparation method and properties of the blends are reported in our previous paper. ${ }^{11}$

\section{Measurement}

DSC: Crystallization kinetics were studied with the following isothermal DSC method (du Pont 910): Samples of about $5 \mathrm{mg}$ were heated to a suitable melt temperature at a scanning rate of $20^{\circ} \mathrm{C} \mathrm{min}^{-1}$ and kept at this temperature for complete melting of crystals (to insure destruction of any active nuclei), and then cooled rapidly to the crystallization temperature. As soon as the samples reached the crystallization temperature, the crystallization exotherm was recorded. The total area under the crystallization exotherm, $\Delta H_{\infty}$, corresponded to the observed heat of crystallization. The fractional crystallization at time $t, X_{t}$, was determined by dividing the area under the crystallization exotherm from the beginning of crystallization to time $t, \Delta H_{t}$ by the total area, $\Delta H_{\infty}$. All the DSC runs were made under a nitrogen atmosphere.

Optical Microscopy: The optical texture of polymers in the liquid crystalline and crystalline states was observed by a polarizing microscope (Leitz, Ortholux) with a hot stage (Mettler FP-2) attachment. The hot stage was controlled by a digital program controller with a built-in microprocessor (REX-P100). The magnification of all micrographs was $320 \mathrm{X}$.

\section{Avrami Analysis}

The generally accepted theory, proposed by Avrami, ${ }^{12,13}$, relates the uncrystallized fraction, $1-X_{t}$, to time, $t$,

$$
-\ln \left(1-X_{t}\right)=K_{n} t^{n}
$$

where $K_{n}$ is the overall rate constant and $n$ is the Avrami exponent which indicates the geometry of the crystal growth process (Table I). The crystallization kinetic data will be in the form of the fractional crystallization as a function of time. To analyze such data it is convenient to take the logarithm of both sides 
Table I. Avrami exponent-sporadic nucleation

\begin{tabular}{cl}
\hline$n$ Value & \multicolumn{1}{c}{ Type of growth } \\
\hline 2 & Linear (1-dimensional) \\
3 & Plate-like (2-dimensional) \\
4 & Spherical (3-dimensional) \\
\hline
\end{tabular}

of the above equation.

$$
\ln \left(-\ln \left(1-X_{t}\right)\right)=\ln \left(K_{n}\right)+n \ln (t)
$$

According to this equation, a plot of $\ln \left(-\ln \left(1-X_{t}\right)\right)$ versus $\ln (t)$ will have a slope of $n$. The overall rate constant, $K_{n}$, can be determined from the slope, $n$, and the half time for the complete crystallization, $t_{1 / 2}$.

$$
K_{n}=\ln 2 /\left(t_{1 / 2}\right)^{n}
$$

The overall rate constant will vary with the crystallization temperature.

\section{RESULTS AND DISCUSSION}

\section{Homopolymers}

Irrespective of the crystallization temperature, the crystallization kinetics of the polymers follow the Avrami equation up to high degree of crystallinity. Typical examples of the Avrami plot of the SP10 homopolymer at different crystallization temperatures are illustrated in Figure 1, in which a linear trend is observed. The kinetic parameters of the NP6 and SP10 homopolymers obtained from the Avrami analysis are listed in Tables II and III, respectively. Since the NP10 homopolymer showed multiple crystallization exotherms, useful crystallization kinetic data were not obtained.

As shown in Table II, the average observed value of the Avrami exponent ( $n$ ) for NP6 is 2.21 , which indicates that the material is in a one-dimensional or rod-like crystallization mode (Table I). The micrographs shown in Figure 2 help to verify this kinetic analysis. The micrographs were taken under a crossed nicol on the same area before and after crystal-

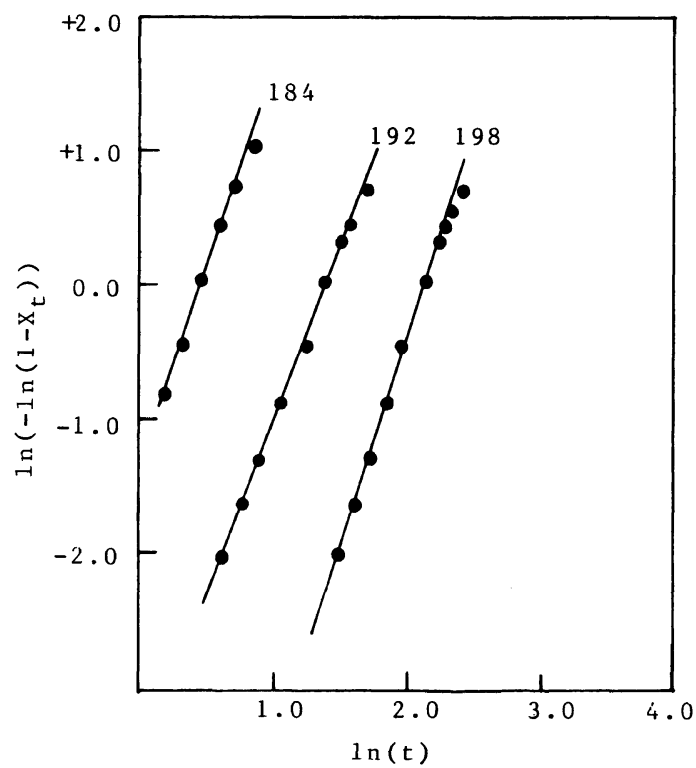

Figure 1. Typical examples of Avrami plot of SP10 at different crystallization temperatures: cryst. temp is given in ${ }^{\circ} \mathrm{C}$, time in $\mathrm{s}$.

Table II. Kinetic parameters of NP6 obtained

\begin{tabular}{|c|c|c|c|}
\hline$T_{\mathrm{c}}$ & $t_{1 / 2}$ & & $K_{n}$ \\
\hline${ }^{\circ} \mathrm{C}$ & $\min$ & & $\min ^{-n}$ \\
\hline 208 & 0.50 & 2.24 & 3.27 \\
\hline 210 & 0.58 & 2.18 & 2.27 \\
\hline 212 & 0.75 & 1.98 & 1.22 \\
\hline 214 & 1.05 & 2.04 & $6.27 \times 10^{-1}$ \\
\hline 216 & 1.13 & 2.32 & $5.22 \times 10^{-1}$ \\
\hline 218 & 1.53 & 2.45 & $2.44 \times 10^{-1}$ \\
\hline
\end{tabular}
by Avrami analysis

Table III. Kinetic parameters of SP10 obtained

\begin{tabular}{|c|c|c|c|}
\hline$T_{\mathrm{c}}$ & $t_{1 / 2}$ & \multirow{2}{*}{$n$} & $K_{n}$ \\
\hline${ }^{\circ} \mathrm{C}$ & $\min$ & & $\min ^{-n}$ \\
\hline 184 & 1.33 & 3.01 & $2.93 \times 10^{-1}$ \\
\hline 186 & 1.58 & 3.11 & $1.67 \times 10^{-1}$ \\
\hline 188 & 1.93 & 3.17 & $8.62 \times 10^{-2}$ \\
\hline 190 & 2.38 & 3.02 & $5.05 \times 10^{-2}$ \\
\hline 192 & 3.15 & 2.83 & $2.69 \times 10^{-2}$ \\
\hline 194 & 4.17 & 2.92 & $1.07 \times 10^{-2}$ \\
\hline 196 & 4.80 & 2.89 & $7.45 \times 10^{-3}$ \\
\hline 198 & 6.50 & 3.22 & $1.67 \times 10^{-3}$ \\
\hline
\end{tabular}
by Avrami analysis 


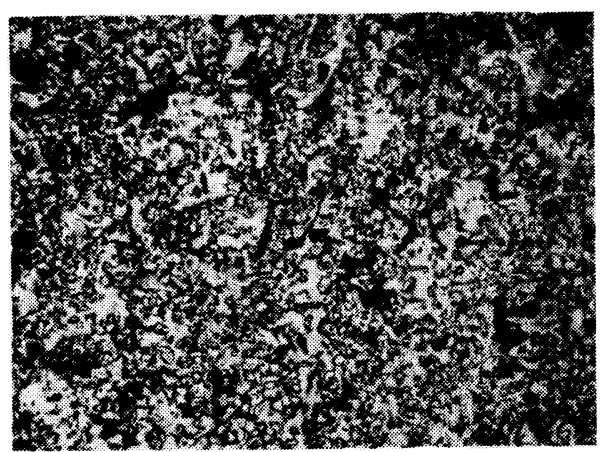

(a)

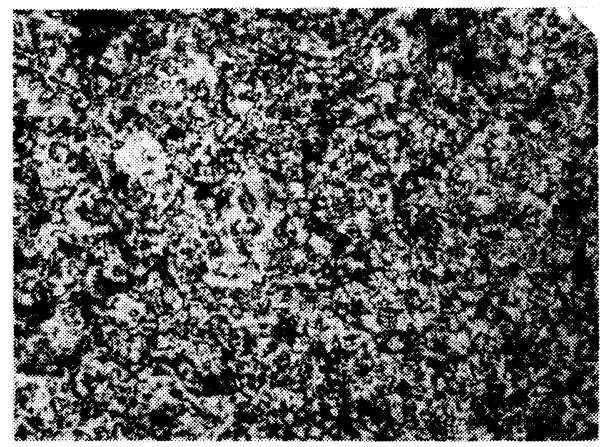

(b)

Figure 2. Textures of NP6 homopolymers: (a) at $250^{\circ} \mathrm{C}$ (nematic); (b) at $218^{\circ} \mathrm{C}$ (solid crystal).

lization: the upper micrograph (Figure 2(a)) was taken at $250^{\circ} \mathrm{C}$ (nematic state) and the lower micrograph (Fig. 2(b)) was taken after the temperature was lowered to $218^{\circ} \mathrm{C}$ at a rate

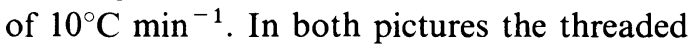
texture characteristic of the nematic mesophase could be observed. From these results, it was found that there is very little reorganization during the crystallization accompanied with only a very small entropy change. In this respect the crystallization behavior of NP6 resembles that of the rigid mesogenic polymers investigated by Warner and $\mathrm{Jaffe}^{6}{ }^{6}$ although the present polymer contains a hexamethylene spacer in the main chain.

Unlike NP6, in the SP10 homopolymer, gross reorganization was observed during crystallization. In Table III, the Avrami exponent is about 3, which corresponds to the two-

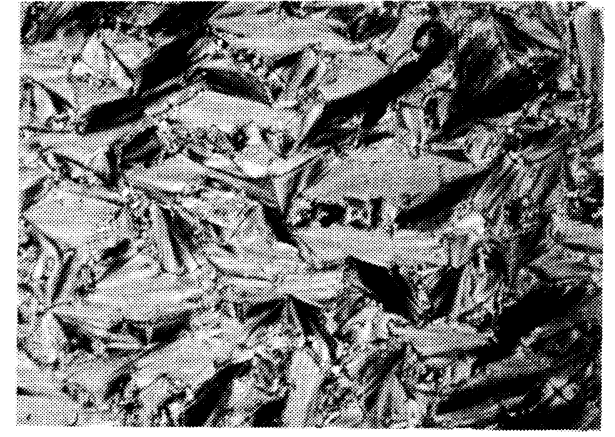

(a)

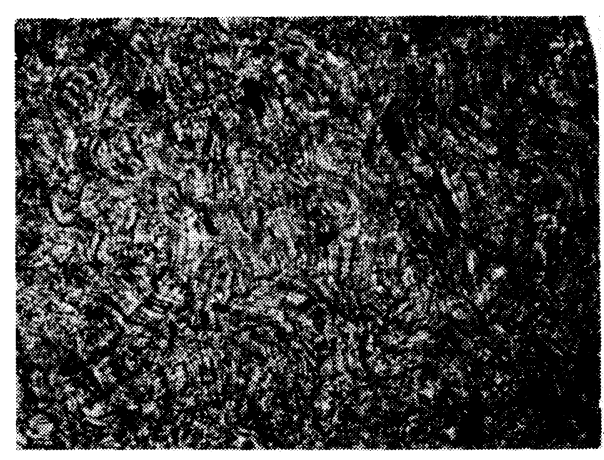

(b)

Figure 3. Textures of SP10 homopolymers: (a) at $250^{\circ} \mathrm{C}$ (smectic); (b) at $185^{\circ} \mathrm{C}$ (solid crystal).

dimensional or plate-like growth from sporadic nuclei (Table I). Microscopic observation of the polymer (Figure 3) showed that the structure of solid state was very different from that of the smectic melt. NP10 homopolymer which formed a nematic state in the melt also showed similar behavior (Figure 4) to that of SP10. This indicates that some extent of structural reorganization may also occur in the crystallization of NP10, although detailed kinetic data of this homopolymer are not available. The crystallization behavior of SP10 and NP10 homopolymers, which is different from that of NP6, is possibly due to the longer flexible spacer. From these observations, it may be concluded that the crystallization of the semi-flexible liquid crystalline polymers is significantly affected by the flexible spacer length. Values of heat of crystallization $\left(\Delta H_{c}\right)$ 


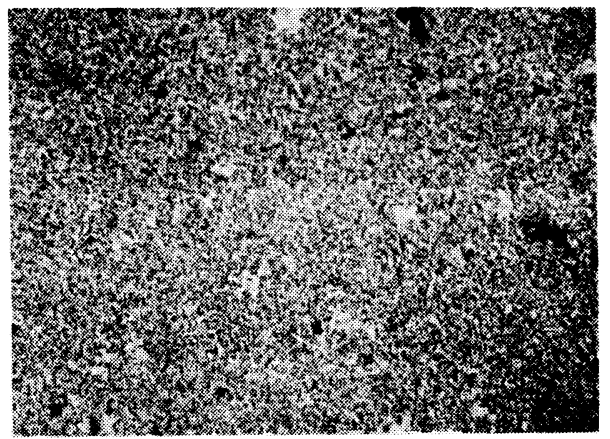

(a)

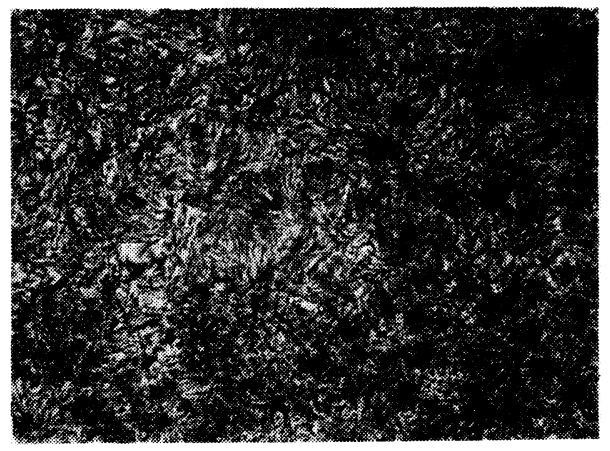

(b)

Figure 4. Textures of NP10 homopolymers: (a) at $250^{\circ} \mathrm{C}$ (nematic); (b) at $200^{\circ} \mathrm{C}$ (solid crystal).

should also give an indication of such crystallization behavior. The values of $\Delta H_{\mathrm{c}}$ for the homopolymers containing decamethylene spacer (SP10, NP10) were significantly larger than that of NP6, being are 3.9, 4.3, and $0.9 \mathrm{kcal}$ $\mathrm{mol}^{-1}$, respectively.

Figure 7 shows the effect of the crystallization temperature on the crystallization rate of NP6 and SP10 as measured by the half-time of complete crystallization, $t_{1 / 2}$, which is also listed in Tables II and III. From the Figure, two points are indicated: (1) the crystallization of SP10 occurs more slowly compared to that of NP6 and (2) the crystallization rate of SP10 is strongly affected by the crystallization temperature, while that of NP6 is relatively insensitive. The overall rate constant $K_{n}$, as shown in the Tables, shows the same trend. It is interesting to note that in spite of the highly ordered smectic structure in the melt, ready to crystallize rapidly, the crystallization of SP10 occurs more sluggishly than that of NP6. The massive rearrangement during crystallization and highly viscous nature of the smectic polymer must be the major contributing factors for lessening the crystallization rate.

The crystallization of all the polymers discussed above, however, occurs much more rapidly compared to ordinary semicrystalline polymers. For example, the half-time of PET ranges from 11.5 to $26.5 \mathrm{~min}$ depending on the crystallization temperature. ${ }^{14}$ This is possibly dut to the fact that the liquid crystalline polymers have ordered structures in their melt state, and the crystallization from the liquid crystalline state occurs rapidly compared to that from the random coil state. Further investigation must be made to clarify the extent to which the results obtained can be generalized for other liquid crystalline polymers.

\section{Blends}

The objective of this investigation was to obtain information on the crystallization behavior of the smectic and nematic polymer blends. Figure 5 shows the typical DSC thermograms of the NP10-SP10 blend with various compositions. These thermograms were obtained by the following standard procedure: The samples were heated to 20 degrees above the melting point to ensure complete melting (in liquid crystalline state) and kept at this temperature for $5 \mathrm{~min}$. Thereafter the samples were cooled at a scanning rate of $10^{\circ} \mathrm{C} \mathrm{min}^{-1}$.

The exothermic peak temperatures in DSC (referred to as the crystallization peak temperature) of NP10, NP6 and SP10 in the blends measured from the DSC curves are plotting against the composition in Figure 6. The Figure shows that the presence of a second component significantly affects the rate of crystallization of the other component. For the SP10 component, the crystallization peak temperatures first increased with increasing con- 


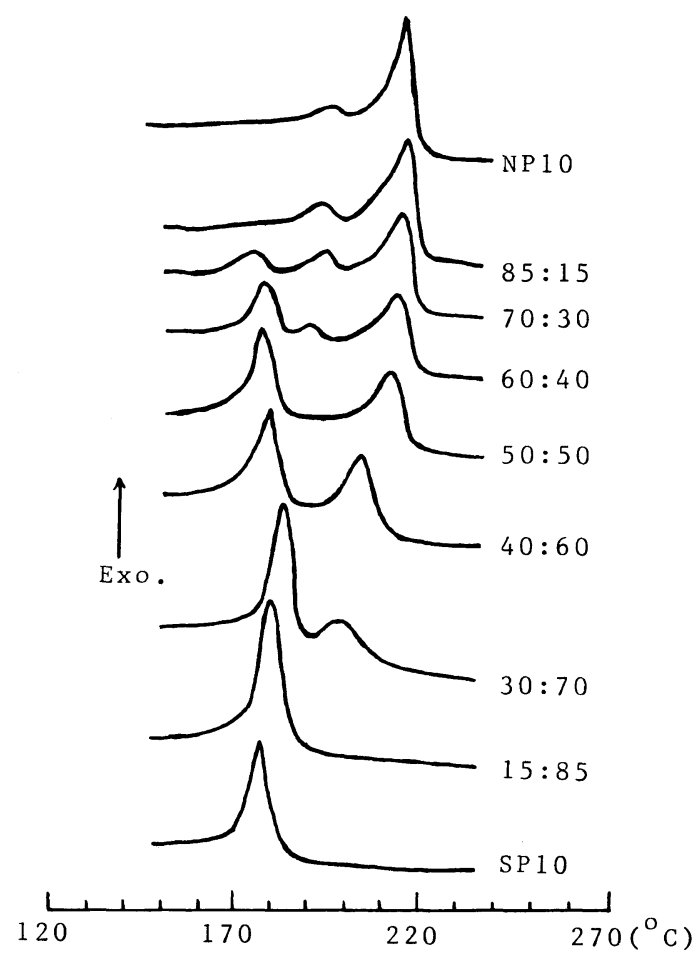

Figure 5. Typical DSC curves of NP10-SP10 blend at various compositions (cooling at $10^{\circ} \mathrm{C} / \mathrm{min}$ ). centration of the nematic component, reached a maximum at a composition around $30 \%$ of NP10 or $15 \%$ of NP6, and thereafter started to decrease. In contrast to this, the crystallization peak temperature of the nematic component steadily decreased as the concentration of SP10 increased.

It is interesting to note from the Figure that the peak crystallization temperature of SP10 component in the NP6-SP10 blend was lower than that in the NP10-SP10 blend. This may be possibly due to the large difference of viscosity of the two nematic homopolymers, and the melt viscosity of NP6 is very high compared to that of NP10. The viscosity values of the two polymers, measured by the Rheometrics Dynamic Spectrometer (RDS$7700)$ at $250^{\circ} \mathrm{C}, 100 \mathrm{rad} \mathrm{s}^{-1}$, were $9.1 \times 10^{2}$ for NP6 and $2.4 \times 10$ Poise for NP10. The viscosity of SP10 at the same condition was $1.3 \times$ $10^{3}$. Poise. The actual melt viscosity data are reported in ref 15 . This highly viscous melt of NP6 hinders the diffusion of the smectic molecules. At flow concentration of either the smectic or nematic component a single homo-

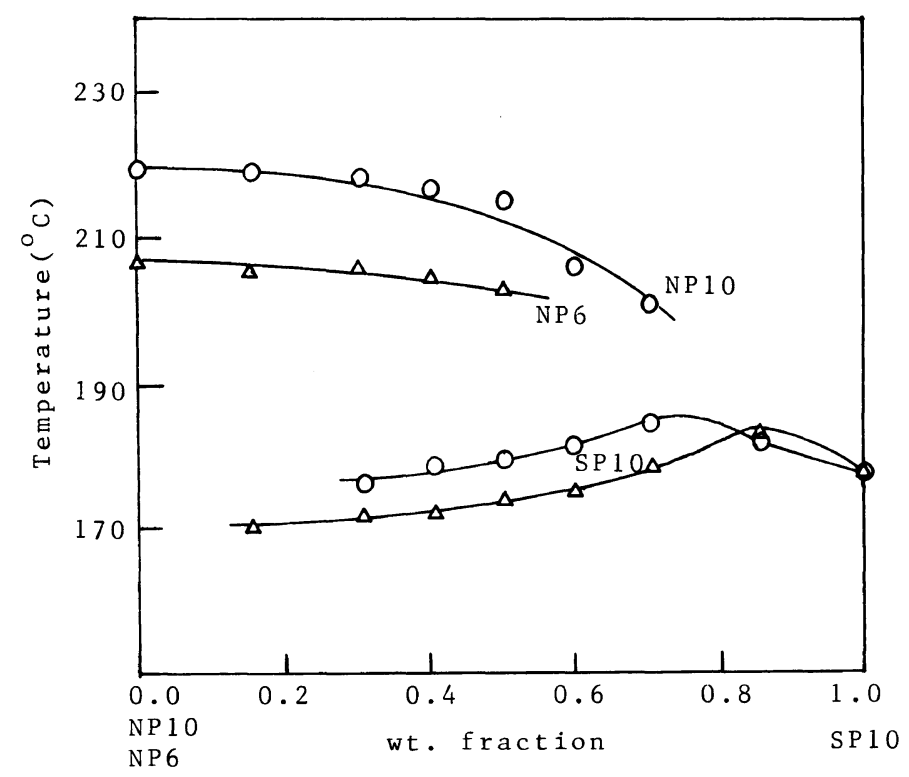

Figure 6. Variation in crystallization peak temperature of homopolymers in the blends: $(O)$ NP10-SP10 blend; $(\triangle)$ NP6-SP10 blend. 
geneous nematic or smectic phase was observed in the phase diagrams, ${ }^{11}$ and a single crystallization exotherm due to the crystallization of the major component was observed. It seems that in this region the crystallization of the minor component is greatly inhibited by the major component, and can not be detected by DSC, even though an extremely small amount of the minor component crystal may exist.

It is well known that the rate of polymer crystallization is controlled by two factors, the rate of nucleation and rate of crystal growth, and in the region near the melting point, nucleation controls the overall crystallization

Table IV. Kinetic parameters of SP10 in the NP6-SP10 mixture containing $85 \%$ SP 10

\begin{tabular}{|c|c|c|c|}
\hline$T_{\mathrm{c}}$ & $t_{1 / 2}$ & & $K_{n}$ \\
\hline${ }^{\circ} \mathrm{C}$ & $\min$ & & $\min ^{-n}$ \\
\hline 189 & 0.70 & 2.91 & 1.96 \\
\hline 190 & 0.76 & 3.02 & 1.59 \\
\hline 191 & 0.97 & 3.35 & $7.67 \times 10^{-1}$ \\
\hline 192 & 1.23 & 3.27 & $3.52 \times 10^{-1}$ \\
\hline 193 & 2.03 & 3.27 & $6.84 \times 10^{-2}$ \\
\hline 194 & 2.62 & 3.40 & $2.62 \times 10^{-2}$ \\
\hline 195 & 3.75 & 2.85 & $1.60 \times 10^{-2}$ \\
\hline 196 & 4.83 & 3.12 & $5.09 \times 10^{-3}$ \\
\hline
\end{tabular}

rate. From this consideration, the crystallization behavior of the blends may be interpreted as follows: for the blends rich in SP10, extremely small quantities of the nematic polymer crystal may act as heterogeneous nucleus, hence, the number of nuclei per unit volume (nucleation density) increased with increase in the amount of the second component. This leads to rapid crystallization of SP10 in the blends. But further increase of the second component retards the crystallization of SP10, indicating that the formation of SP10 crystal is strongly disturbed by excessively large amounts of the second components. At these compositions, the nematic and smectic phases co-

Table V. Kinetic parameters of SP10 in the NP6-SP 10 mixture containing 50\% SP10

\begin{tabular}{cccc}
\hline$\frac{T_{\mathrm{c}}}{{ }^{\circ} \mathrm{C}}$ & \multicolumn{1}{c}{$t_{1 / 2}$} & & \multicolumn{1}{c}{$K_{n}$} \\
\cline { 2 - 4 } $\min ^{-n}$ & $\min$ & & 1.87 \\
\hline 179 & 0.72 & 3.02 & $4.34 \times 10^{-1}$ \\
181 & 1.15 & 3.34 & $2.07 \times 10^{-1}$ \\
183 & 1.50 & 2.98 & $9.83 \times 10^{-2}$ \\
185 & 1.87 & 3.12 & $3.12 \times 10^{-2}$ \\
187 & 2.42 & 3.51 & $2.17 \times 10^{-2}$ \\
189 & 3.08 & 3.08 & $3.37 \times 10^{-3}$ \\
191 & 3.93 & 3.32 & $3.94 \times 10^{-3}$ \\
193 & 5.08 & 3.18 & \\
\hline
\end{tabular}

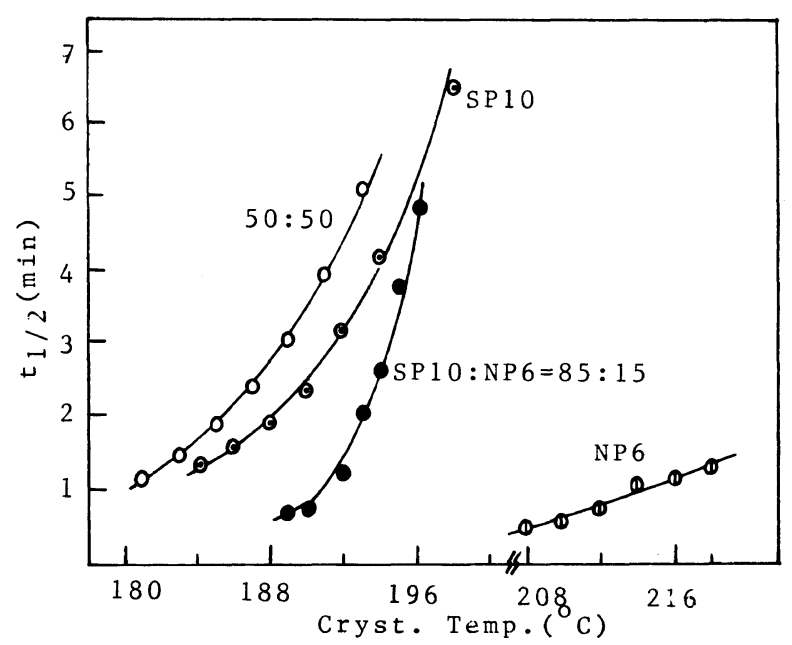

Figure 7. Effect of crystallization temperature on the crystallization rate as measured by the half-time for complete crystallization. 
exist in the liquid crystalline state and thus the nucleating role of the nematic polymer diminished. Unlike the smectic polymer, crystallization rates of the nematic polymers in the blends decrease continuously without such a maximum. This is possibly due to the presence of melt smectic phase in the blends during the crystallization of the nematic polymers. This highly viscous liquid state hinders the diffusion of the nematic molecules, and there may be no increase of the nucleation density.

In order to clarify the crystallization behavior of SP10, the kinetic aspects of the crystallization of SP10 in the NP6-SP10 blend were studied. The results are reported in Tables IV and V. Table IV shows the kinetic parameters for the mixture of $85 \%$ SP 10 , which exhibited a maximum crystallization rate of SP10, and Table $\mathrm{V}$ shows those for a mixture of $50 \%$ SP10, which exhibited a lower crystallization rate than the smectic homopolymer (see Figure 6). The half-times of crystallization of the mixtures are presented in Figure 7 for comparison of both homopolymers. The Figure shows the mixture having $85 \%$ SP10 to crystallize more rapidly than the homopolymer, whereas the crystallization of the $50: 50$ mixture to occur slowly. This is in good agreement with the variation in crystallization peak temperature shown in Figure 6. It is interesting to note from the figure that despite the presence of NP6, the temperature dependence of the crystallization rate of SP10 in the mixtures was quite high as in the case of the smectic homopolymer.

Acknowledgement. The authors wish to acknowledge the financial support for this work from YUKONG LIMITED (formely Korea Oil Corporation).

\section{REFERENCES}

1. P. Adamski and S. Klomczyk, Sov. Phys. Crystallogr., 23, 82 (1978).

2. P. Adamski and R. Czyzewski, Sov. Phys. Crystallogr., 23, 725 (1978).

3. F. P. Price and J. H. Wendorff, J. Phys. Chem., 76, 276 (1972).

4. F. P. Price and A. K. Fritzsche, J. Phys. Chem., 77, 396 (1973).

5. F. P. Price and J. H. Wendorff, J. Phys. Chem., 75, 2839 (1971).

6. S. B. Warner and M. Jaffe, J. Cryst. Growth, 48, 184 (1980).

7. S. Antoun, R. W. Lenz, and J. I. Jin, J. Polym. Sci., Polym. Phys. Ed., 19, 1901 (1981).

8. C. K. Ober, J. I. Jin, and R. W. Lenz, Makromol. Chem., Rapid Commun., 4, 49 (1983).

9. G. Chen and R. W. Lenz, J. Polym. Sci., Polym. Chem. Ed., 22, 3189 (1984).

10. R. W. Lenz, Polym. J., 17, 105 (1985).

11. Y. D. Yoo and S. C. Kim, Polym. Bull., accepted for publication.

12. M. Avrami, J. Chem. Phys., 7, 1103 (1939).

13. M. Avrami, J. Chem. Phys., 8, 212 (1940).

14. D. Garcia, J. Polym. Sci., Polym. Phys. Ed., 22, 2063 (1984).

15. Y. D. Yoo, Ph. D. Thesis, Chap. IV, KAIST (1987). 